\title{
Competing Weak Localization and Weak Antilocalization in Ultrathin Topological Insulators
}

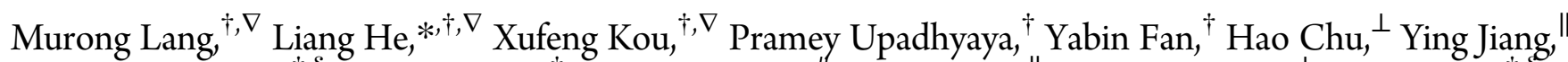 \\ Jens H. Bardarson, ${ }^{\ddagger}$, Wanjun Jiang, ${ }^{\dagger}$ Eun Sang Choi, ${ }^{\#}$ Yong Wang, Nai-Chang Yeh, ${ }^{\perp}$ Joel Moore, ${ }^{\ddagger}, \S^{\prime}$ \\ and Kang L. Wang*, \\ ${ }^{\dagger}$ Department of Electrical Engineering, University of California, Los Angeles, California 90095, United States \\ ${ }^{\ddagger}$ Department of Physics, University of California, Berkeley, California 94720, United States \\ ${ }^{\S}$ Materials Sciences Division, Lawrence Berkeley National Laboratory, Berkeley, California 94720, United States \\ "Center of Electron Microscopy, State Key Laboratory of Silicon Materials, Department of Materials Science and Engineering, \\ Zhejiang University, Hangzhou, 310027, China \\ ${ }^{\perp}$ Department of Physics, California Institute of Technology, Pasadena, California 91125, United States \\ ${ }^{\#}$ National High Magnetic Field Laboratory, Tallahassee, Florida 32310, United States
}

Supporting Information

ABSTRACT: We demonstrate evidence of a surface gap opening in topological insulator (TI) thin films of $\left(\mathrm{Bi}_{0.57} \mathrm{Sb}_{0.43}\right)_{2} \mathrm{Te}_{3}$ below six quintuple layers through transport and scanning tunneling spectroscopy measurements. By effective tuning the Fermi level via gate-voltage control, we unveil a striking competition between weak localization and weak antilocalization at low magnetic fields in nonmagnetic ultrathin films, possibly owing to the change of the net Berry phase. Furthermore, when the Fermi level is swept into the surface gap of ultrathin samples, the overall unitary behaviors are revealed at higher magnetic fields, which are in contrast to the pure WAL signals obtained in thicker films. Our findings show an exotic phenomenon characterizing the gapped TI surface states and point to the future realization of quantum spin Hall effect and dissipationless TI-based applications.

KEYWORDS: Topological insulator, ambipolar effect, surface states hybridization, ultrathin films, weak localization, weak antilocalization

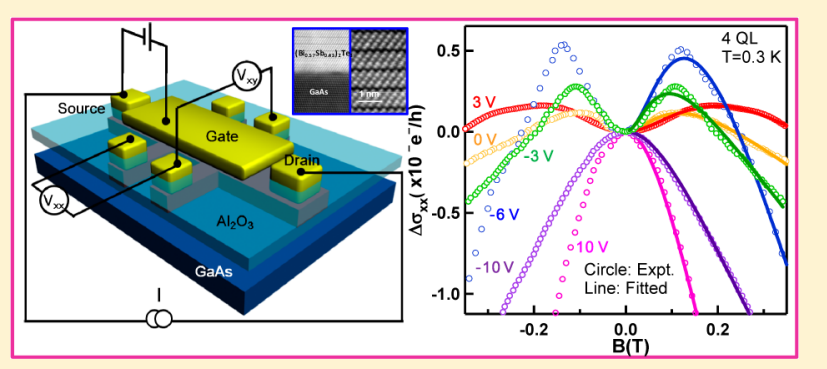

(1)
$\mathrm{T}$ hree-dimensional (3D) topological insulators (TIs) are distinct materials featuring bulk insulting states and unique massless Dirac fermions in the surface state. ${ }^{1-3}$ In particular, the spins of electrons in these states are tightly locked to their momentums due to the strong spin-orbit coupling, and thus backscattering in the Dirac fermions by nonmagnetic impurities is prohibited due to the protection by time-reversal symmetry. ${ }^{4-6}$ As a result, the topological protection of the surface states could potentially be applied for future novel applications, including low-dissipation electronics, interconnect, thermoelectric, quantum computin,g and others. $^{7-13}$

Massless topological surface states have been experimentally identified and extensively studied in binary and ternary TI materials. ${ }^{14-30}$ More recently, the exotic and interesting physics associated with the coupling between the top and the bottom surface states in the two-dimensional (2D) limit of these 3D TIs has attracted new attention. ${ }^{19,31-35}$ However, to date only a few experiments have been carried out on ultrathin TI films, and most of them focus on angle-resolved photoemission spectroscopy ${ }^{19}$ and scanning tunneling spectroscopy (STS). ${ }^{35}$ 
a

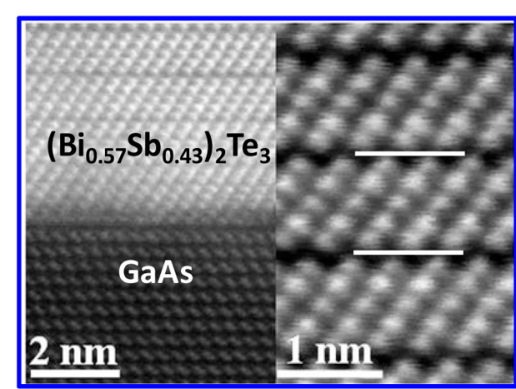

C

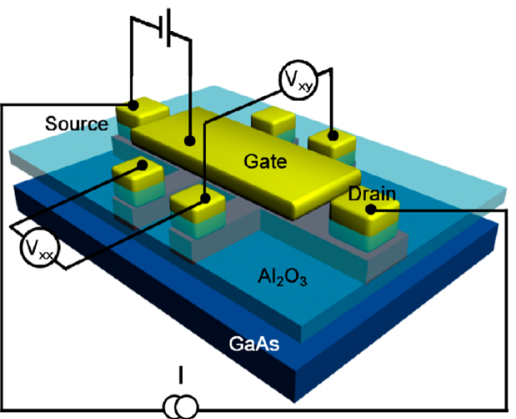

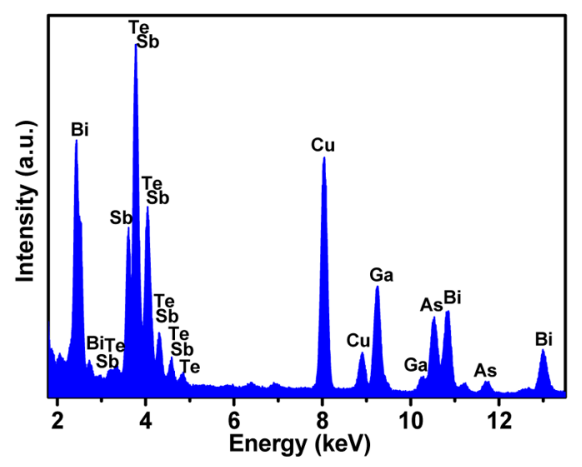

d

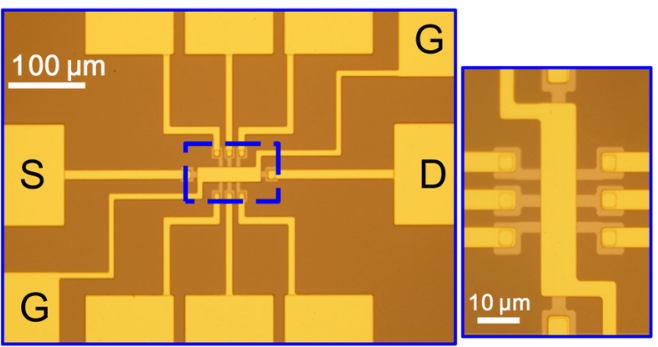

Figure 1. Cross-section TEM, EDX and device structure. (a) Left: A high-resolution TEM picture for the $\left(\mathrm{Bi}_{0.57} \mathrm{Sb}_{0.43}\right)_{2} \mathrm{Te}_{3}$ film and GaAs $(111) \mathrm{B}$ substrate. An atomically sharp interface is also observed. Right: A magnified TEM image showing the quintuple structure with $\sim 1.02 \mathrm{~nm}$ lattice spacing between (0003) planes. (b) Energy dispersive $\mathrm{X}$-ray spectroscopy of the film composition, which is mainly composed of $\mathrm{Bi}$, $\mathrm{Sb}$, and $\mathrm{Te}$. (c) Schematic diagram of $\left(\mathrm{Bi}_{0.57} \mathrm{Sb}_{0.43}\right)_{2} \mathrm{Te}_{3}$ (red) on a highly resistive GaAs (111) (dark blue) top-gate Hall bar device structure with measurement setup. The total thickness of $\mathrm{Al}_{2} \mathrm{O}_{3}$ (light blue) is $\sim 25 \mathrm{~nm}$. A standard four-point measurement was carried out with constant AC current flow of 1 $\mu \mathrm{A}$. (d) Left: An optical image of the device structure with a $100 \mu \mathrm{m}$ scale bar. Right: A zoom-in view of the Hall bar with the size of $5 \mu \mathrm{m}(L) \times 5$ $\mu \mathrm{m}(W)$, with a $10 \mu \mathrm{m}$ scale bar.

to the change of Berry phase, presenting a unique way to study the surface states of the ultrathin TI with a gap opening at the Dirac point in the quantum diffusive regime.

High-quality single crystalline $\left(\mathrm{Bi}_{x} \mathrm{Sb}_{1-x}\right)_{2} \mathrm{Te}_{3}$ thin films were grown on high resistivity $(\approx 10 \mathrm{M} \Omega \cdot \mathrm{cm}) \mathrm{GaAs}(111) \mathrm{B}$ substrates in an ultrahigh vacuum molecular beam epitaxy (MBE) system. In situ real-time reflection high energy electron diffraction (RHEED) was used to monitor the pseudomorphic growth dynamics with the electron beam incidence along the $[11 \overline{2} 0]$ direction (Supporting Information, Figure S1). To investigate the quality of the thin film, high-resolution scanning transmission electron microscope (STEM) experiments were performed on a FEI TITAN Cs corrected STEM operating at $200 \mathrm{kV}$. Figure 1a (left) shows a high angle annular dark field (HAADF) image of the single crystalline $\left(\mathrm{Bi}_{0.53} \mathrm{Bi}_{0.47}\right)_{2} \mathrm{Te}_{3}$ film and the GaAs substrate, where the sharp interface without any amorphous layer is clearly revealed. The QLs of this ternary TI system is further manifested in the magnified TEM image (Figure 1a, right), demonstrating the same tetradymite structure as their parent compounds of $\mathrm{Bi}_{2} \mathrm{Te}_{3}$ and $\mathrm{Sb}_{2} \mathrm{Te}_{3}{ }^{37}$ The thickness of each QL is estimated to be $\sim 1.02 \mathrm{~nm}$, as indicated by two parallel white lines. Additionally, the energy dispersive X-ray spectroscopy (EDX) was performed to examine the film composition. Prominent $\mathrm{Bi}, \mathrm{Sb}$, and $\mathrm{Te}$ peaks are revealed in Figure $1 \mathrm{~b}$. Quantitative analysis shows that the precise atomic ratio of $\mathrm{Bi} / \mathrm{Sb} / \mathrm{Te}$ is $1.35: 1: 3.4$. To explore the transport properties of this material, a top-gate field effect transistor (FET) device was fabricated as schematically shown in Figure 1c (see Supporting Information). Figure 1d (left) shows an optical microscope image of the typical device structure. The diagonal two electrodes are top-gate electrodes, whereas the rest electrodes are connected to the Hall bar. A zoom-in view of the eight-terminal Hall bar $(5 \mu \mathrm{m} / 15 \mu \mathrm{m}$ (length) $\times 5 \mu \mathrm{m}$ (width)) is shown in the right panel.

Magneto-transport measurements were carried out by feeding a constant ac current of $1 \mu \mathrm{A}$ at $13 \mathrm{~Hz}$ at a base temperature of $0.3 \mathrm{~K}$. A characteristic ambipolar field effect is observed in thin films with thickness ranging from 4 to $9 \mathrm{QL}$ as displayed in Figure $2 \mathrm{a}-\mathrm{f}$. By applying a top gate bias $V_{\mathrm{g}}, E_{\mathrm{F}}$ can be effectively tuned across the surface Dirac point, and both the carrier type as well as the carrier density can be easily controlled. Remarkably, a giant gate modulation of $\sim 1500 \%$ with a sharp peak resistance $\left(R_{\max }\right)$ of $\sim 70 \mathrm{k} \Omega$ is observed in the ultrathin 4 QL film (Figure 2a, upper panel). Compared with the gate modulations in the thicker samples shown in Figure $2 c-f$ (upper panels), this high on/off ratio can be attributed to the extreme low density of states in the $4 \mathrm{QL}$ sample as a result of surface gap opening. Furthermore, the Hall coefficient $\left(R_{\mathrm{H}}\right)$ (open squares) extracted from the Hall slope reverses its sign when $R_{x x}$ approaches its maximum, corresponding to the lowest net carrier density achieved. Alternatively, the $2 \mathrm{D}$ carrier density $n_{\mathrm{H}}=-1 /\left(e R_{\mathrm{H}}\right)$ (where $e$ is the elementary charge) is calculated to further illustrate this ambipolar effect, as indicated in Figure 2a (lower panel). Here, the unipolar conduction (only one type of dominant carrier) is manifested in the linear $p$-type $\left(V_{\mathrm{g}}<-3 \mathrm{~V}\right)$ and $n$-type region $\left(V_{\mathrm{g}}>5 \mathrm{~V}\right)$, whereas in the ambipolar region $\left(-3 \mathrm{~V}<V_{\mathrm{g}}<5 \mathrm{~V}\right)$, $R_{\mathrm{H}}$ crosses zero and $n_{\mathrm{H}}$ changes sign from $p$ to $n$-type, suggesting the coexistence of two competing carriers (electron and hole). ${ }^{37,38}$

For the $5 \mathrm{QL}$ sample (Figure 2b), the resistance peak broadens, and $R_{\max }$ decreases to $\sim 14 \mathrm{k} \Omega$ due to a smaller gap opening. Moreover, $R\left(V_{\mathrm{g}}\right)$ shows a much reduced modulation signature of only $\sim 250 \%$. As the film thickness further increases (Figure $2 \mathrm{c}-\mathrm{f}$, upper panel), $R\left(V_{\mathrm{g}}\right)$ exhibits a much weaker 
a
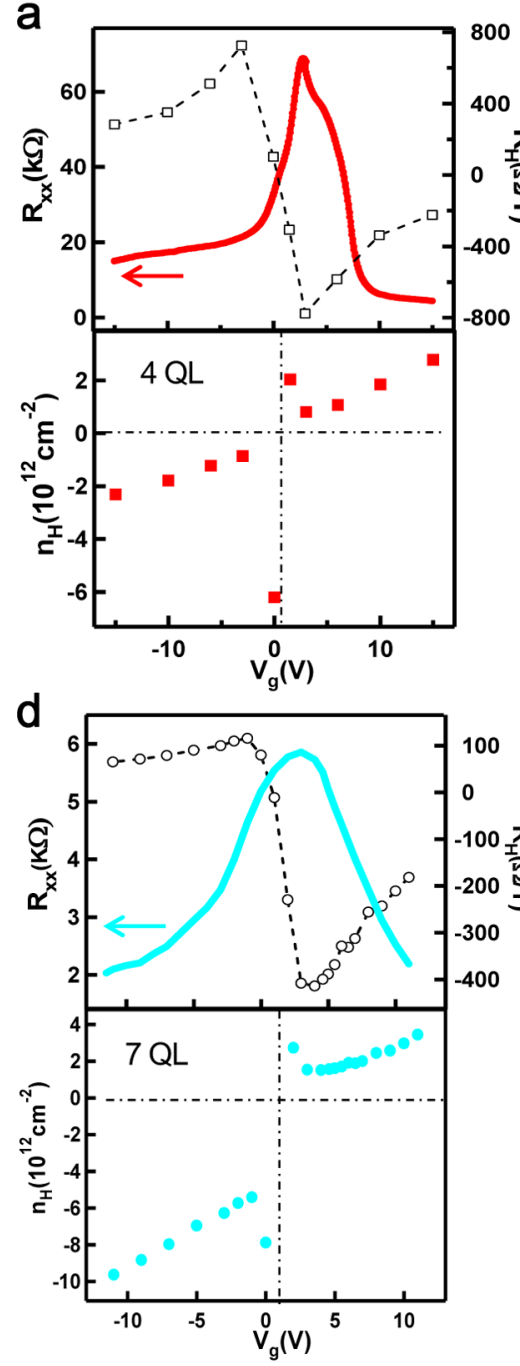

b
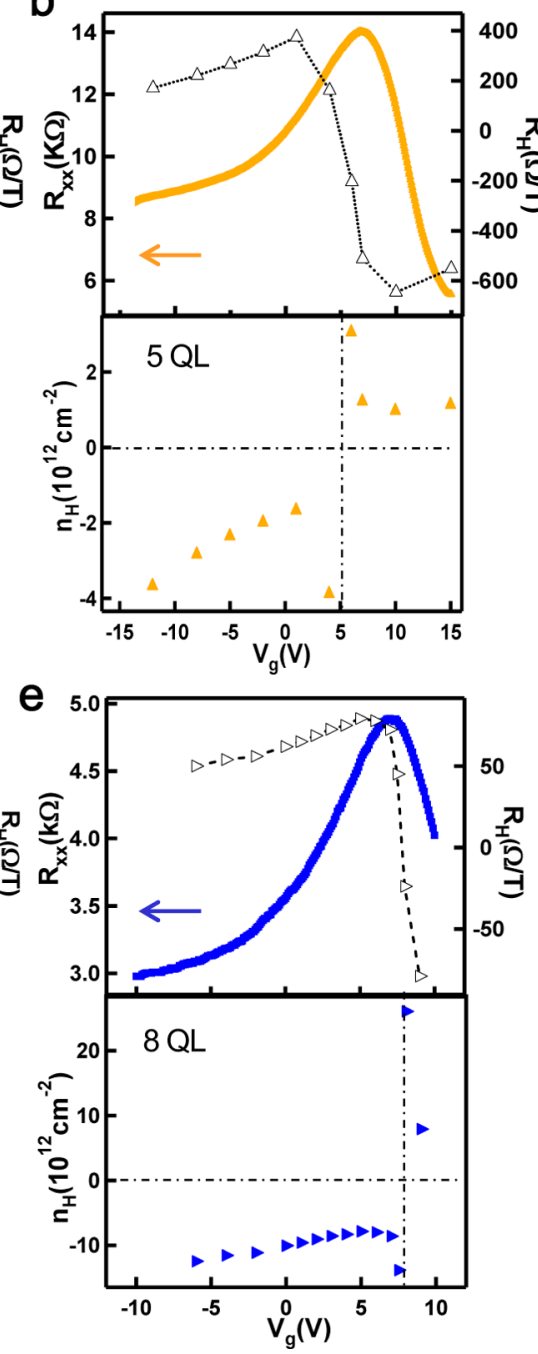
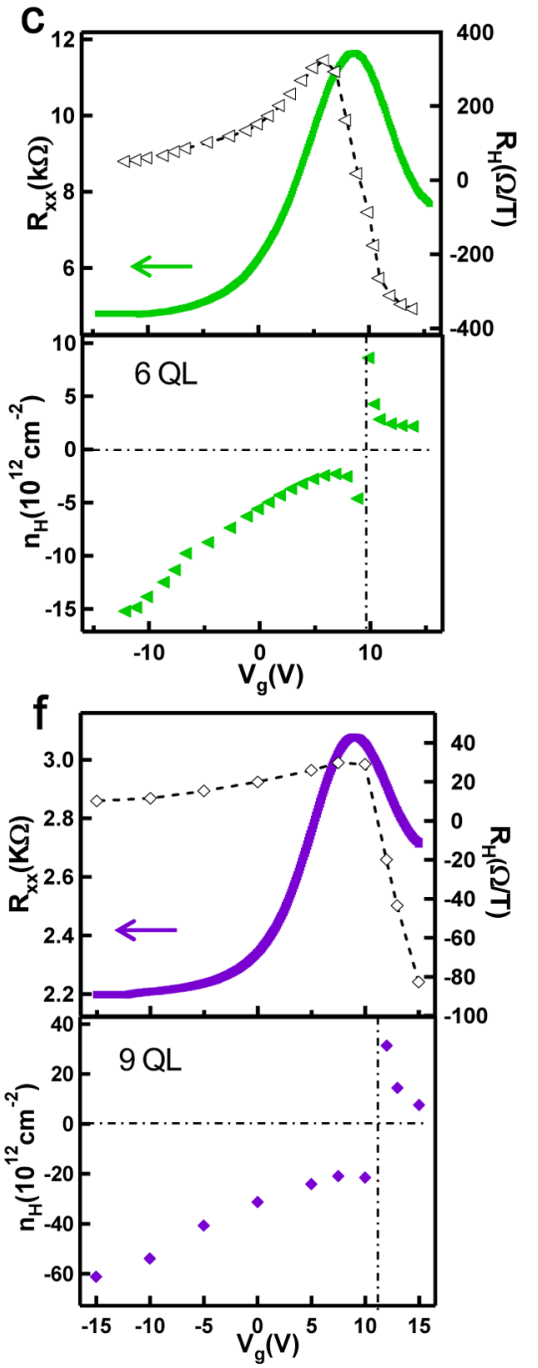

Figure 2. Transport properties of thickness dependent $\left(\mathrm{Bi}_{0.57} \mathrm{Sb}_{0.43}\right)_{2} \mathrm{Te}_{3}$ at $0.3 \mathrm{~K}$. (a-f) Upper: Longitudinal resistance $R_{x x}$ (lines) and Hall coefficient $R_{\mathrm{H}}$ (open symbols) as functions of gate voltage $V_{\mathrm{g}}$ corresponding to different thicknesses from 4 to 9 QLs. The largest peak of $R_{x x}$ vs $V_{\mathrm{g}}$ and giant gate modulation of $\sim 1500 \%$ are observed in the 4 QL sample. As the film thickness increases, the peak broadens and $R_{\max }$ decreases greatly. Each $R_{\mathrm{H}}$ is extracted from the Hall traces at $\pm 4 \mathrm{~T}$ for a certain $V_{\mathrm{g}}$. Lower: Unipolar conduction (only one type of dominant carrier) is manifested in the linear regions of $n_{\mathrm{H}}$ vs $V_{\mathrm{g}}$. While in the ambipolar region, $n_{\mathrm{H}}$ starts to diverge at the bias (changing sign from $\mathrm{p}$ to $\mathrm{n}$ ), where the net carrier density is significantly reduced, indicating the ambipolar behavior.

tunability, and $R_{\max }$ decreases its magnitude, indicating the disappearance of the surface hybridized gap and an increased bulk denisties. In particular, $R_{\max }$ of the 9 QL film is suppressed by 1 order of magnitude relative to that of the $4 \mathrm{QL}$ film, accompanied by an increase of minimum carrier density $n_{\min }$ from $\sim 8 \times 10^{11} \mathrm{~cm}^{-2}$ (4 QL) to $1 \times 10^{13} \mathrm{~cm}^{-2}$ (9 QL). Remarkably, a sudden rise of $n_{\min }$ at thickness $t>7$ QL may be attributed to the appearance of bulk conduction, which could no longer be fully depleted by gating due to the relatively short depletion width in TI materials (Supporting Information, Figure S3). ${ }^{33,37}$

The ambipolar effect presented above reveals a giant gate modulation and an ultralow carrier density in 4 QL film when $E_{\mathrm{F}}$ is tuned through the hybridization induced surface gap. Next, we present the striking influence of the surface gap on the magneto-transport properties of the ultrathin TI films in the quantum diffusive region. Figure $3 a$ displays the dramatic contrast of normalized magnetoconductivity (MC) $\Delta \sigma(B)=$ $\sigma_{x x}(B)-\sigma_{x x}(0)$ between thick $(6,7,9,10 \mathrm{QL})$ and thin $(4$ and $5 \mathrm{QL}$ ) films at their $V_{\text {Dirac }}$ (the $V_{\mathrm{g}}$ corresponding to $R_{\max }$ ) as a function of perpendicular external magnetic field applied at $T=$ $0.3 \mathrm{~K}$. On one hand, for $t \geq 6 \mathrm{QL}$, all of the MC curves display a sharp negative cusp characteristic of WAL, consistent with the nontrivial topology of the surface states carrying a Berry phase of $\pi .^{20,22,23,32,39}$ The data can be analyzed via the HikamiLarkin-Nagaoka (HLN) formula below: ${ }^{40}$

$$
\Delta \sigma=\frac{\alpha e^{2}}{2 \pi^{2} \hbar}\left[\psi\left(\frac{l_{B}^{2}}{l_{\phi}^{2}}+\frac{1}{2}\right)-\ln \left(\frac{l_{B}^{2}}{l_{\phi}^{2}}\right)\right]
$$

where $\psi$ is the digamma function; the prefactor $\alpha$ can be used as an estimate of the number of independent channels contributing to the interference, $\alpha=-1 / 2$ for a single channel WAL and $\alpha=-1$ for two channels; and $l_{\phi}$ is the phase coherence length. As indicated in the inset of Figure $3 a$, the fitting results yield $|\alpha| \approx 1$ for the thicker films, suggesting the coexistence of two fully decoupled channels in this system, contributing $|\alpha|=1 / 2$ each. ${ }^{41,42}$ The slight increase of $|\alpha|$ as film gets thicker, is possibly owing to the additional bulk channel 

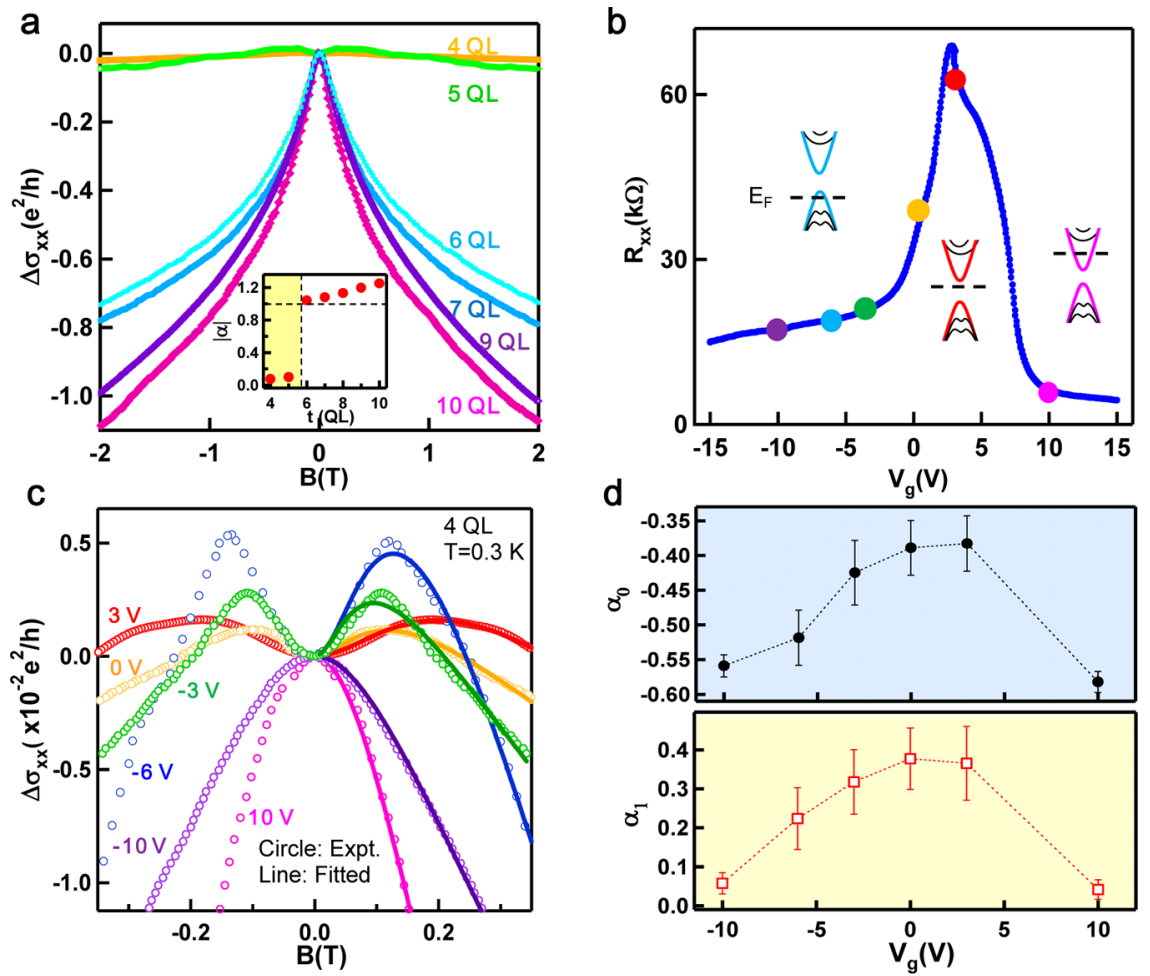

Figure 3. Quantum interference competition in $4 \mathrm{QL}\left(\mathrm{Bi}_{0.57} \mathrm{Sb}_{0.43}\right)_{2} \mathrm{Te}_{3}$ at $0.3 \mathrm{~K}$. (a) Normalized magnetoconductance $(\mathrm{MC}) \Delta \sigma(B)=\sigma_{x x}(B)-$ $\sigma_{x x}(0)$ for $4-10$ QL measured at their $V_{\text {Dirac }}$. The conductivity is significantly suppressed when the thickness is reduced to 4 and 5 QLs. Inset: thickness dependent $\alpha$ fitted from one component HLN theory shows an abrupt change as $t<6 \mathrm{QL}$. (b) Gate voltage dependence of resistance of the $4 \mathrm{QL}$ sample. The dots on the curve represent the specific gate biases applied for each corresponding magnetoresistance measurements in part $\mathrm{c}$. The insets show schematics of $E_{\mathrm{F}}$ positions relative to the gapped surface states. (c) Evolution of low field MC as a function of gate voltage for the 4 QL film. In the ambipolar region $\left(V_{\mathrm{g}}=-6,-3,0,3 \mathrm{~V}\right)$, the MC curves first show WL-like behavior at low field $(B<0.2 \mathrm{~T})$ and then bend over to WAL at higher field. The MC recovers their WAL characteristics again when $E_{\mathrm{F}}$ is high or low enough to be in the unipolar region $\left(V_{\mathrm{g}}=-10,10 \mathrm{~V}\right)$. (d) The evolution of $\alpha_{0}$ (upper panel) and $\alpha_{1}$ (lower panel) fitted by eq 2 as functions of gate voltage. The two competing WAL and WL behaviors coexist and compete with each other in the ambipolar region.

partially coupled with the surfaces. ${ }^{41,42}$ On the other hand, consistent with ref 33, when the film thickness is reduced to the 2D limit (4 and 5 QLs), the MC curves at their $V_{\text {Dirac }}$ change drastically from the sharp WAL behavior to a unitary behavior $(\alpha \approx 0)$, suggesting the opening of a surface gap in 2D limit TIs. It should be emphasized that for the gap-opened 2D limit of TIs eq 1 is no longer valid and quantum correction to MC can acquire both WAL and WL contributions, which, for the case of weak surface and bulk coupling, acquires a modified HLN equation given in eq 2 , to be discussed later. We further note that in contrast to the study in ref 33 that was limited to a fixed Fermi level, our current approach of controlling the Fermi level via gating provides further confirmation and a more complete picture of the combined WAL and WL contributions to MC for massive Dirac fermions, which we elaborate below.

Strikingly, at low magnetic fields $(B<0.4 \mathrm{~T})$, we observe an intriguing electric field controlled quantum interferences competition between WAL and WL effect as shown in Figure $3 \mathrm{c}$ for the $4 \mathrm{QL}$ film and Figure S4a for the $5 \mathrm{QL}$ one (Supporting Information, Figure S5), both of which exhibit marked differences from thicker ones. For the $4 \mathrm{QL}$ film, in the ambipolar region $\left(-6 \mathrm{~V} \leq V_{\mathrm{g}} \leq 3 \mathrm{~V}\right)$, when $E_{\mathrm{F}}$ is inside/close to the surface gap, the MC first exhibits a WL-like behavior at low field $(B<0.2 \mathrm{~T})$, and then bends over to WAL at higher field. We also note that at $V_{\mathrm{g}}=3 \mathrm{~V}$, where $R_{\max }$ is obtained, the weakest magnetic field dependence of MC (unitary-like behavior) is achieved, implying the absence of interference because of an insulating state. However, as $E_{\mathrm{F}}$ is moved deep into the unipolar region $\left(V_{\mathrm{g}}=10,-10 \mathrm{~V}\right)$, the WL signal vanishes completely, and the MC curves recover the WAL with negative cusps, similar to pure Dirac-like surface states. The band structure for the gapped surface state with respect to their $E_{\mathrm{F}}$, is schematically shown in the inset of Figure $3 \mathrm{~b}$ in three different gate voltage regimes.

In particular, we extract the relative strength of WAL and WL contributions as a function of gate voltage by fitting the measured MC curves to the two-component HLN theory: ${ }^{42}$

$$
\Delta \sigma(B)=\sum_{i=0,1} \frac{\alpha_{i} e^{2}}{\pi h}\left[\psi\left(\frac{l_{B}^{2}}{l_{\phi i}^{2}}+\frac{1}{2}\right)-\ln \left(\frac{l_{B}^{2}}{l_{\phi i}^{2}}\right)\right]
$$

Here, the coefficients $\alpha_{0}$ and $\alpha_{1}$ in eq 2 stand for the weights of WAL and WL contributions from the two surfaces, respectively, and $l_{\phi i}$ is the corresponding phase coherence length. The WAL dominated MC ideally has prefactors $\alpha_{0}=-1 / 2$ and $\alpha_{1}=0$, while the WL dominated MC leads to $\alpha_{0}=0$ and $\alpha_{1}=1 / 2$. Equation 2 gives excellent fit to all the gate voltage dependent $\mathrm{MC}$ curves in Figure $3 \mathrm{c}$ as shown by solid lines. Figure $3 \mathrm{~d}$ summarizes the evolution of $\alpha_{0}$ and $\alpha_{1}$ of the $4 \mathrm{QL}$ sample as functions of applied gate voltage. When $V_{\mathrm{g}}$ moves positively or negatively away from $V_{\text {Dirac }}\left|\alpha_{0}\right|$ increases while $\alpha_{1}$ decrease, indicating the WL contribution is greatly suppressed, whereas the WAL contribution becomes more pronounced. Specifically, at $V_{\mathrm{g}}=10 \mathrm{~V}$, as $E_{\mathrm{F}}$ lies high into the upper branch of surface 

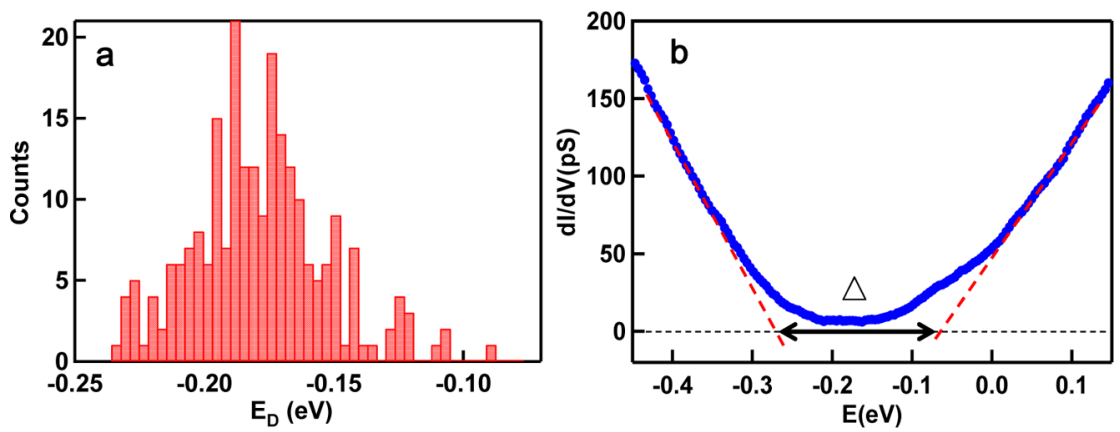

Figure 4. Scanning tunneling spectroscopy of $4 \mathrm{QL}\left(\mathrm{Bi}_{0.57} \mathrm{Sb}_{0.43}\right)_{2} \mathrm{Te}_{3}$. (a) The histogram of Dirac energy $\left(E_{\mathrm{D}}\right)$ estimated from the midgap energy to be $E_{\mathrm{D}}=-0.17 \pm 0.07 \mathrm{eV}$ throughout the areas of our investigation, and the relative large variation is probably due to the surface terraces. (b) A typical tunneling conductance $(\mathrm{d} I / \mathrm{d} V)$ spectrum taken at $77 \mathrm{~K}$, showing a surface bandgap opening in the 4 QL thin film. Here the small tunneling conductance in the gap region may be attributed to the thermal smearing effect.

state where a $\pi$ Berry phase is recovered, WAL dominates the MC curve with negligible WL component $\left(\alpha_{0}=-0.582 \pm\right.$ 0.015 , and $\alpha_{1}=0.0412 \pm 0.025$ ), and a similar scenario can also be seen in the other end of the bias $\left(V_{\mathrm{g}}=-10 \mathrm{~V}\right)$. On the contrary, $\mathrm{WL}$ contribution is maximized while WAL is minimized $\left(\alpha_{0}=-0.383 \pm 0.039\right.$ and $\left.\alpha_{1}=0.365 \pm 0.095\right)$ as $V_{\mathrm{g}}$ approaches $V_{\text {Dirac }}=3 \mathrm{~V}$.

The observation of WL in the ultrathin films is surprising. In fact, for an ideal TI film without bulk conduction in the diffusive regime, WAL is obtained in a numerical simulation. ${ }^{43,44}$ It has been suggested that a WL contribution can be obtained from the bulk if it is weakly doped and decoupled from the surface. ${ }^{41}$ However, our result does not seem to be consistent with a bulk contribution because the bulk contribution is greatly suppressed in ultrathin films when $E_{\mathrm{F}}$ is near the surface bandgap. Alternatively, this observed behavior can be understood in terms of Berry phase $(\phi)$ acquired by electrons over closed trajectories, which is dependent on gate voltage for gapped Dirac fermions, in the form of $\phi=\pi\left(1-\Delta /\left(2 E_{\mathrm{F}}\right)\right)$ (see also Supporting Information, Figure S7). ${ }^{45}$ When $E_{\mathrm{F}}$ is close to the Dirac point, $\phi$ approaches 0 and therefore results in the dominance of WL. Tuning $E_{\mathrm{F}}$ from $V_{\text {Dirac }}$ deeper into the surface band takes $\phi$ from 0 to $\pi$, which one might expect to correspond with the transition from WL to WAL. ${ }^{42,45,46}$ However, one should remember that there are two Dirac cones and the spinor corresponding to the top/ bottom index can play an important role. Nevertheless, since $E_{\mathrm{F}}$ of the top and bottom surface is different due to the band bending, the Berry phase reduction mentioned above might still be relevant. ${ }^{37}$ This requires further theoretical exploration and is beyond the scope of this work.

Moreover, we also observe the electric field controlled WAL/ $\mathrm{WL}$ quantum interference competition in the $5 \mathrm{QL}$ film as manifested in Figure S4a. We further remark that the WAL behaviors reported in the literature for samples $t \leq 5 \mathrm{QL}$ is consistent with the condition $E_{\mathrm{F}}{ }^{2} \gg \Delta^{2}$, suggesting that the chemical potential of those samples typically lies far outside the surface state. $^{32,33}$ On the contrary, for thicker films ( $\geq 6$ QL), WAL clearly manifests itself in various gate voltages, consistent with the absence of surface gap, as shown in the Supporting Information, Figure S5.

To determine the Dirac energy and surface bandgap opening in the ultrathin $4 \mathrm{QL}\left(\mathrm{Bi}_{0.57} \mathrm{Sb}_{0.43}\right)_{2} \mathrm{Te}_{3}$ sample, low-temperature spatially resolved STS under high vacuum was carried out. A detailed survey of the tunneling conductance spectra was acquired over a $10 \mathrm{~nm} \times 10 \mathrm{~nm}$ area with $64 \times 64$ pixels at 77
$\mathrm{K}$. The Dirac energy $\left(E_{\mathrm{D}}\right)$ estimated from the midgap energy is $E_{\mathrm{D}}=-0.17 \pm 0.07 \mathrm{eV}$ throughout the areas of our investigation as shown by the histogram in Figure 4 a. Figure $4 b$ presents a typical tunneling conductance $(\mathrm{d} I / \mathrm{d} V)$ spectrum that manifests a surface hybridization bandgap $\sim 180 \mathrm{meV}$ in the 4 QL sample.

In conclusion, we have demonstrate the ambipolar field effect of electrically surface manipulation in high quality and low carrier density MBE-grown $\left(\mathrm{Bi}_{0.57} \mathrm{Sb}_{0.43}\right)_{2} \mathrm{Te}_{3}$ thin films. Due to the hybridization-induced surface gap, the $\mathrm{MC}$ of 4 and $5 \mathrm{QL}$ ultrathin films are greatly suppressed, exhibiting overall unitarylike behavior when $E_{\mathrm{F}}$ is located inside the surface bandgap. By tuning $E_{\mathrm{F}}$ position relative to the gap, the striking crossover between WL and WAL is observed in nonmagnetic ultrathin films at low field region, a characteristic feature of quantum interferences competition. Hence, our observation is of great importance for further understanding the role of surface state hybridization in ultrathin TIs, and these hybridized surface states can be promising for the realization of edge states for demonstrating the quantum spin Hall effect and dissipationless spintronics in 3D TIs.

\section{ASSOCIATED CONTENT}

\section{Supporting Information}

MBE growth of $\left(\mathrm{Bi}_{0.57} \mathrm{Sb}_{0.43}\right)_{2} \mathrm{Te}_{3}$, sample characterization methods, device fabrication process, maximum resistance and 2D carrier density vs thickness, quantum interferences competition in 5 QL samples, weak antilocalization in 6-10 QLs and theoretical calculation of two parameters $\alpha_{0}$ and $\alpha_{1}$. This material is available free of charge via the Internet at http://pubs.acs.org.

\section{AUTHOR INFORMATION}

\section{Corresponding Author}

*E-mail: wang@ee.ucla.edu, heliang@ee.ucla.edu.

\section{Author Contributions}

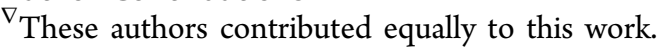

\section{Notes}

The authors declare no competing financial interest.

\section{ACKNOWLEDGMENTS}

The authors acknowledge helpful discussions with R. Mong from UC Berkeley and technical support from X. Yu, M. Wang, J. Tang, and L. Chang from the Device Research Laboratory at UCLA. This work was in part supported by Defense Advanced Research Projects Agency (DARPA), Focus Center Research Program-Center on Functional Engineered Nano Architec- 
tonics (FENA). A portion of this work was performed at the National High Magnetic Field Laboratory, which is supported by NSF Cooperative Agreement No. DMR-0654118, by the State of Florida, and by the Department of Energy (DOE). Y.W. acknowledges the support from Natural Science Foundation of China (11174244) and Zhejiang Provincial Natural Science Foundation of China (LR12A04002) and National Young 1000 Talents Plan.

\section{REFERENCES}

(1) Hasan, M. Z.; Kane, C. L. Rev. Mod. Phys. 2010, 82 (4), 3045.

(2) Moore, J. E. Nature 2010, 464 (7286), 194-198.

(3) Qi, X.-L.; Zhang, S.-C. Rev. Mod. Phys. 2011, 83 (4), 1057-1110.

(4) Qi, X.-L.; Hughes, T. L.; Zhang, S.-C. Phys. Rev. B 2008, 78 (19), 195424.

(5) Kane, C. L.; Mele, E. J. Phys. Rev. Lett. 2005, 95 (14), 146802.

(6) Moore, J. E.; Balents, L. Phys. Rev. B 2007, 75 (12), 121306.

(7) Fu, L.; Kane, C. L. Phys. Rev. Lett. 2008, 100 (9), 096407.

(8) Teweldebrhan, D.; Goyal, V.; Balandin, A. A. Nano Lett. 2010, 10 (4), 1209-1218.

(9) Nayak, C.; Simon, S. H.; Stern, A.; Freedman, M.; Das Sarma, S. Rev. Mod. Phys. 2008, 80 (3), 1083-1159.

(10) Moore, J. Nat. Phys. 2009, 5 (6), 378-380.

(11) Goyal, V. Appl. Phys. Lett. 2010, 97 (13), 133117.

(12) Zahid, F.; Lake, R. Appl. Phys. Lett. 2010, 97 (21), 212102.

(13) Teweldebrhan, D.; Goyal, V.; Rahman, M.; Balandin, A. A. Appl. Phys. Lett. 2010, 96 (5), 053107-3.

(14) Hsieh, D.; Qian, D.; Wray, L.; Xia, Y.; Hor, Y. S.; Cava, R. J.; Hasan, M. Z. Nature 2008, 452 (7190), 970-974.

(15) Xia, Y.; Qian, D.; Hsieh, D.; Wray, L.; Pal, A.; Lin, H.; Bansil, A.; Grauer, D.; Hor, Y. S.; Cava, R. J.; Hasan, M. Z. Nat. Phys. 2009, 5 (6), $398-402$.

(16) Kong, D.; Dang, W.; Cha, J. J.; Li, H.; Meister, S.; Peng, H.; Liu, Z.; Cui, Y. Nano Lett. 2010, 10 (6), 2245-2250.

(17) Butch, N. P.; Kirshenbaum, K.; Syers, P.; Sushkov, A. B.; Jenkins, G. S.; Drew, H. D.; Paglione, J. Phys. Rev. B 2010, 81 (24), 241301.

(18) Analytis, J. G.; McDonald, R. D.; Riggs, S. C.; Chu, J.-H.; Boebinger, G. S.; Fisher, I. R. Nat. Phys. 2010, 6 (12), 960-964.

(19) Zhang, Y.; He, K.; Chang, C.-Z.; Song, C.-L.; Wang, L.-L.; Chen, X.; Jia, J.-F.; Fang, Z.; Dai, X.; Shan, W.-Y.; Shen, S.-Q.; Niu, Q.; Qi, X.-L.; Zhang, S.-C.; Ma, X.-C.; Xue, Q.-K. Nat. Phys. 2010, 6 (8), 584588.

(20) Liu, M.; Chang, C.-Z.; Zhang, Z.; Zhang, Y.; Ruan, W.; He, K.; Wang, L.-l.; Chen, X.; Jia, J.-F.; Zhang, S.-C.; Xue, Q.-K.; Ma, X.; Wang, Y. Phys. Rev. B 2011, 83 (16), 165440.

(21) Lang, M.; He, L.; Xiu, F.; Yu, X.; Tang, J.; Wang, Y.; Kou, X.; Jiang, W.; Fedorov, A. V.; Wang, K. L. ACS Nano 2012, 6, 295-302.

(22) Chen, J.; He, X. Y.; Wu, K. H.; Ji, Z. Q.; Lu, L.; Shi, J. R.; Smet, J. H.; Li, Y. Q. Phys. Rev. B 2011, 83 (24), 241304.

(23) Chen, J.; Qin, H. J.; Yang, F.; Liu, J.; Guan, T.; Qu, F. M.; Zhang, G. H.; Shi, J. R.; Xie, X. C.; Yang, C. L.; Wu, K. H.; Li, Y. Q.; Lu, L. Phys. Rev. Lett. 2010, 105 (17), 176602.

(24) Hsieh, D.; Xia, Y.; Qian, D.; Wray, L.; Dil, J. H.; Meier, F.; Osterwalder, J.; Patthey, L.; Checkelsky, J. G.; Ong, N. P.; Fedorov, A. V.; Lin, H.; Bansil, A.; Grauer, D.; Hor, Y. S.; Cava, R. J.; Hasan, M. Z. Nature 2009, 460 (7259), 1101-1105.

(25) Chen, Y. L.; Analytis, J. G.; Chu, J.-H.; Liu, Z. K.; Mo, S.-K.; Qi, X. L.; Zhang, H. J.; Lu, D. H.; Dai, X.; Fang, Z.; Zhang, S. C.; Fisher, I. R.; Hussain, Z.; Shen, Z.-X. Science 2009, 325 (5937), 178-181.

(26) Xiu, F.; He, L.; Wang, Y.; Cheng, L.; Chang, L.-T.; Lang, M.; Huang, G.; Kou, X.; Zhou, Y.; Jiang, X.; Chen, Z.; Zou, J.; Shailos, A.; Wang, K. L. Nat. Nanotechnol. 2011, 6 (4), 216-221.

(27) Ren, Z.; Taskin, A. A.; Sasaki, S.; Segawa, K.; Ando, Y. Phys. Rev. B 2010, 82 (24), 241306.

(28) He, L.; Xiu, F.; Yu, X.; Teague, M.; Jiang, W.; Fan, Y.; Kou, X.; Lang, M.; Wang, Y.; Huang, G.; Yeh, N.-C.; Wang, K. L. Nano Lett. 2012, 12 (3), 1486-1490.
(29) Wang, Y.; Xiu, F.; Cheng, L.; He, L.; Lang, M.; Tang, J.; Kou, X.; Yu, X.; Jiang, X.; Chen, Z.; Zou, J.; Wang, K. L. Nano Lett. 2012, 12 (3), 1170-1175.

(30) Steinberg, H.; Laloë, J. B.; Fatemi, V.; Moodera, J. S.; JarilloHerrero, P. Phys. Rev. B 2011, 84 (23), 233101.

(31) Cho, S.; Butch, N. P.; Paglione, J.; Fuhrer, M. S. Nano Lett. 2011, 11 (5), 1925-1927.

(32) Kim, Y. S.; Brahlek, M.; Bansal, N.; Edrey, E.; Kapilevich, G. A.; Iida, K.; Tanimura, M.; Horibe, Y.; Cheong, S.-W.; Oh, S. Phys. Rev. B 2011, 84 (7), 073109.

(33) Taskin, A. A.; Sasaki, S.; Segawa, K.; Ando, Y. Phys. Rev. Lett. 2012, 109 (6), 066803.

(34) Zhang, L.; Hammond, R.; Dolev, M.; Liu, M.; Palevski, A.; Kapitulnik, A. arXiv:1205.5832v1, 2012.

(35) Teague, M. L.; Chu, H.; Xiu, F. X.; He, L.; Wang, K. L.; Yeh, N. C. Solid State Commun. 2012, 152 (9), 747-751.

(36) Zhang, J.; Chang, C.-Z.; Zhang, Z.; Wen, J.; Feng, X.; Li, K.; Liu, M.; He, K.; Wang, L.; Chen, X.; Xue, Q.-K.; Ma, X.; Wang, Y. Nat. Commun. 2011, 2, 574.

(37) Kong, D.; Chen, Y.; Cha, J. J.; Zhang, Q.; Analytis, J. G.; Lai, K.; Liu, Z.; Hong, S. S.; Koski, K. J.; Mo, S.-K.; Hussain, Z.; Fisher, I. R.; Shen, Z.-X.; Cui, Y. Nat. Nanotechnol. 2011, 6 (11), 705-709.

(38) Hong, S. S.; Cha, J. J.; Kong, D.; Cui, Y. Nat. Commun. 2012, 3, 757.

(39) He, H.-T.; Wang, G.; Zhang, T.; Sou, I.-K.; Wong, G. K. L.; Wang, J.-N.; Lu, H.-Z.; Shen, S.-Q.; Zhang, F.-C. Phys. Rev. Lett. 2011, 106 (16), 166805.

(40) Hikami, S.; Larkin, A. I.; Nagaoka, Y. Prog. Theor. Phys. 1980, 63 (2), 707-710.

(41) Garate, I.; Glazman, L. Phys. Rev. B 2012, 86 (3), 035422.

(42) Lu, H.-Z.; Shen, S.-Q. Phys. Rev. B 2011, 84 (12), 125138.

(43) Mong, R. S. K.; Bardarson, J. H.; Moore, J. E. Phys. Rev. Lett. 2012, 108 (7), 076804.

(44) Ringel, Z.; Kraus, Y. E.; Stern, A. Phys. Rev. B 2012, 86 (4), 045102 .

(45) Lu, H.-Z.; Shi, J.; Shen, S.-Q. Phys. Rev. Lett. 2011, 107 (7), 076801 .

(46) Ghaemi, P.; Mong, R. S. K.; Moore, J. E. Phys. Rev. Lett. 2010, 105 (16), 166603. 